\title{
The NOCTURNAL Ambient Assisted Living System
}

\author{
W. Carswell, J. Augusto, M. Mulvenna, J. Wallace, \\ S. Martin P.J. McCullagh, H. Zheng, H. Wang \\ School of Computing and Mathematics \\ University of Ulster \\ Northern Ireland \\ \{w.carswell, jc.augusto, md.mulvenna\}@ulster.ac.uk
}

\author{
K. McSorley, B. Taylor, W.P. Jeffers \\ Fold Housing Association \\ Northern Ireland \\ [kevinmcs,barbara.taylor,Paul.Jeffers]@foldgroup.co.uk
}

\begin{abstract}
We describe the NOCTURNAL system which provides Ambient Assisted Living with a focus on night-time support. This paper explains the design principles, the technological infrastructure and the software intelligence which facilitates autonomous assistance in the form of therapeutic interventions.
\end{abstract}

Keywords-Ambient Assisted Living, night-time care, therapeutic interventions, sensing, multi-agent systems.

\section{INTRODUCTION}

The provision of Pervasive Healthcare is attracting significant attention from a wide range of healthcare organizations. This interest is focused by demographics, the impact of demographics on healthcare economics and a desire from people to preserve their independence for longer. However, most of the contributions reported in the technical literature focus on the most active period of the day encompassing the hours of daylight. Our project, NOCTURNAL (Night Optimised Care Technology for UseRs Needing Assisted Lifestyles), is predicated on evidence from the research literature that the night period has specific characteristics which require separate analysis and technological solutions [1]. This paper explains the technological infrastructure of NOCTURNAL, as well as the design and development principles used on the deployment of this solution.

\section{SYSTEM DESIGN}

The NOCTURNAL system is designed to address areas of concern for the service provider as collected from their clients. These areas of concern for night care are diverse but at these initial stages of service development we are focusing on the detection of restlessness, bed occupancy and wandering and their associated therapeutic interventions. Restlessness during sleep is the first situation of concern, followed by the client leaving their bed and then moving around inside their residence, or even outside it (see Figure 1).

The strategy is to consider monitoring in levels of increasing importance the further away from bed in distance and time. Therapeutic interventions are triggered in the same order.

\section{IMPLEMENTATION}

The system technological infrastructure is based on $\mathrm{X} 10$ technology which provides easy deployment at an affordable price for more people. We use pressure mats in bed, PIR sensors and lighting control. These allow us to monitor restlessness in bed and bed occupancy, movement in different rooms of the house and light control. This basic sensing and actuation platform is supplemented by a more versatile interface implemented on a tablet PC (see Figure 2). The technological infrastructure described above provides a flexible, yet affordable, sensing/actuation platform [2].

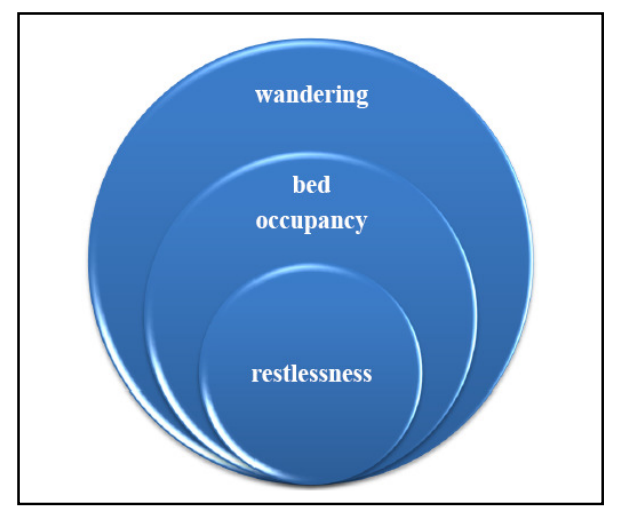

Figure 1. The strict containment of areas indicate the hierarchical relationship between situations of concern. The intervention strategy reflects this relationship.

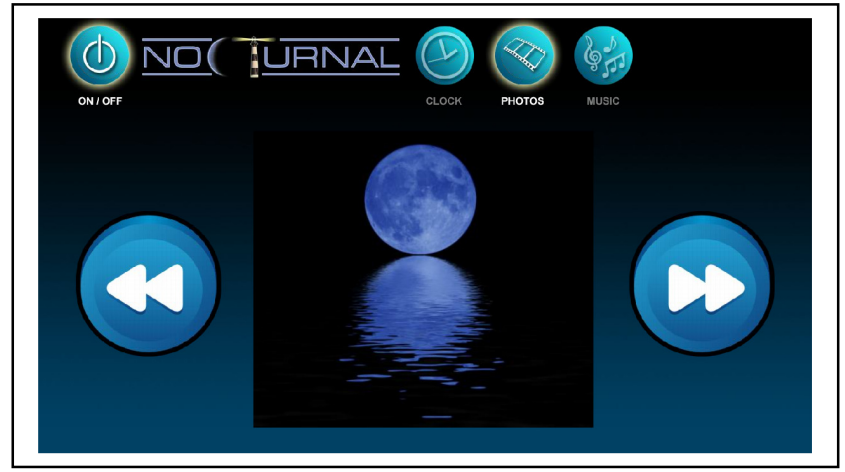

Figure 2. A tablet PC is used to provide soothing images and music and to interact with the clients delivering therapeutical interventions when sitautions of restlessness, bed occupancy and wandering are detected.

At the core of the system there is a multi-agent system which monitors the environment trying to detect any of the following situations: restlessness, bed occupancy and wandering). The system has a dedicated agent monitoring each of those 
situations. If the defining elements of any of those are identified by an agent then a message is sent to a coordinating agent that has an overall view and can decide whether the situation has been correctly identified. If the coordinating agent has good reasons to believe that one of those situations is developing it will send a message to the Therapeutic Intervention Agent (TIA) to trigger the corresponding intervention plan. The success of the intervention will be measured by the changes in behaviour of the client which is sensed in real time, monitored by the specialized agents and reported to the coordinating agent (see Figure 3).

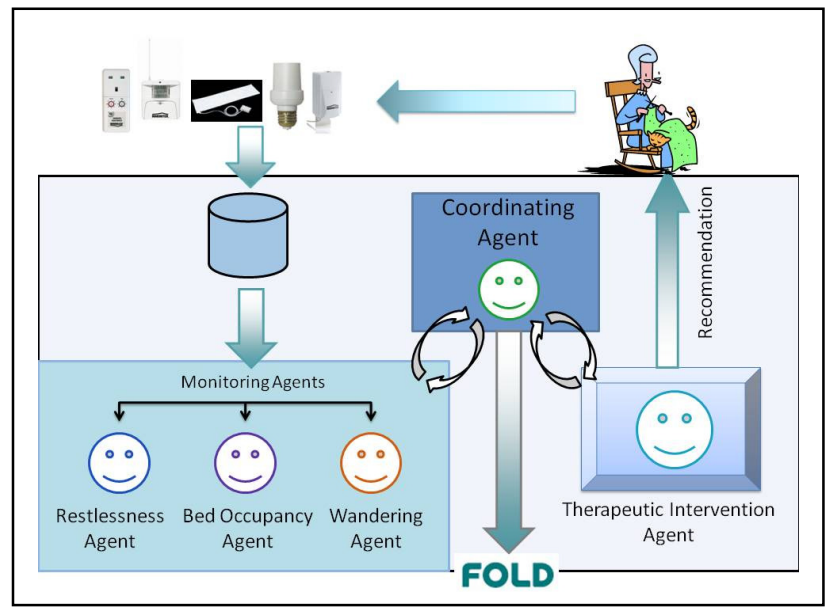

Figure 3. NOCTURNAL multi-agent system architecture.

Figure 4 shows the multi-agent system in action. The agent specialized on detecting restlessness events communicates with the Coordinating Agent who orders the TIA agent to take action. This includes agreed actuations like playing soothing music. The messages below illustrate the second time they attempt this therapy.

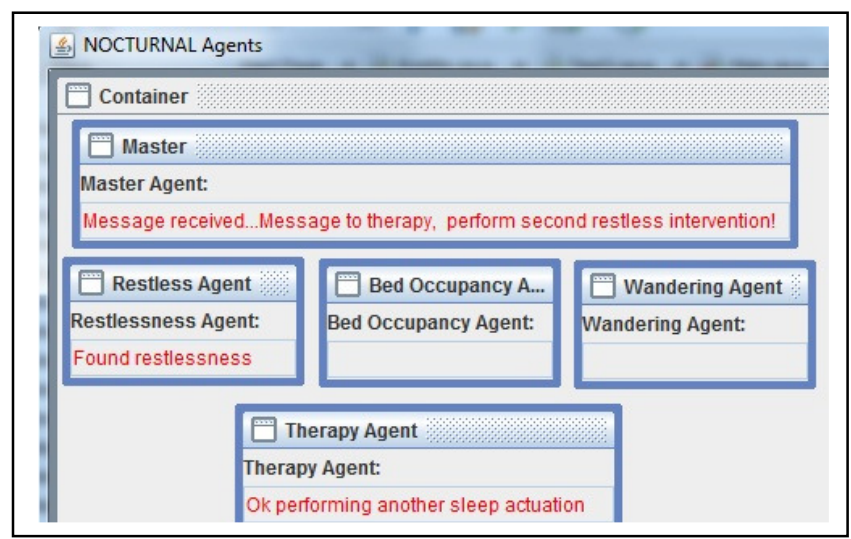

Figure 4. Restlessness detected.

If the restlessness therapy does not work and the client goes out of bed, a different agent will detect this (see Figure 5). In this case other actuations may become relevant as dimming lights to reduce risk of falling and showing messages in the tablet PC which can increase the level of understanding of the situation and attracting the person back to a safer situation.

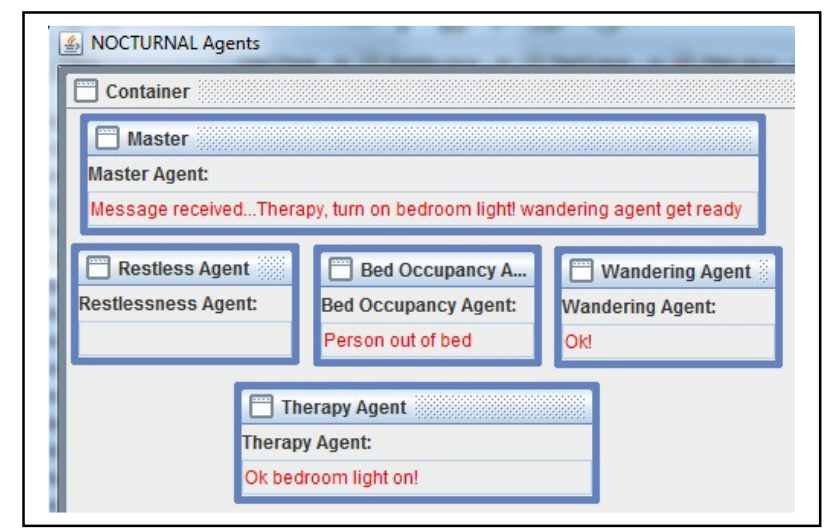

Figure 5. An episode of client getting out of bed is detected.

\section{CONCLUSIONS}

The NOCTURNAL system provides night-time support for people with dementia. Previous developments in the area had focused on isolated factors or dimensions of the problem, using only one sensor (e.g., pressure pad), considering only one area of the house (e.g., bed) or condition to be monitored (e.g. restlessness). NOCTURNAL provides a more holistic approach with a combination of sensors and devices covering a whole house and several important situations of concern.

We designed the system around the idea of concentric areas of increasing concern to monitor and a corresponding therapeutic intervention strategy. The technological infrastructure allows sophisticated sensing and actuation at an affordable price. The development of the system is based on an intelligent multiagent system which associates agents with monitoring and intervention tasks.

\section{REFERENCES}

[1] W. Carswell, P.J. McCullagh, J.C. Augusto, S. Martin, M.D. Mulvenna, H. Zheng, H.Y. Wang, J.G. Wallace, K. McSorley, B. Taylor, W.P. Jeffers, "A Review of the Role of Assistive Technology for People with Dementia in the Hours of Darkness". Technology and Health Care, Volume 17, Number 4, pp. 281-304. 2009. IOS Press.

[2] P.J. McCullagh, W. Carswell, M.D. Mulvenna, J.C. Augusto, H. Zheng, and W.P. Jeffers. "Nocturnal Sensing and Intervention for Assisted Living of People with Dementia.". In Healthcare Sensor Networks Challenges Towards Practical Application. In D. Lai, R. Begg and M. Palaniswami (Eds.). Taylor and Francis/CRC Press. July 2011.

[3] J. C. Augusto, H. Zheng, M. Mulvenna, H. Wang, W. Carswell, P. Jeffers. "Design and Modelling of the Nocturnal AAL Care System". Proceedings of $2^{\text {nd }}$ International Symposium on Ambient Intelligence (ISAmI 2011), April, 2011 - Salamanca - Spain. Springer Verlag.

[4] H. Wang, H. Zheng, J. C. Augusto, S. Martin, M. Mulvenna, W. Carswell, J. Wallace, P. Jeffers, B. Taylor, and K. McSorley. "Monitoring and Analysis of Sleep Pattern for People with Early Dementia". 1st Workshop on Knowledge Engineering, Discovery and Dissemination in Health (KEDDH'10), in Proceedings of 2010 IEEE International Conference on Bioinformatics and Biomedicine Workshops, 18-21 December 2010, Hong Kong, pp. 405 - 410, China. 